\title{
Теоретическая оценка характеристик селективности газохроматографических неподвижных фаз
}

\author{
Зайцева Е.А., Долгоносов А.М. \\ Институт геохимии и аналитической химии им. В.И. Вернадского Российской академии наук \\ (ГЕОХИ РАН), Москва
}

Поступила в редакцию 11.07.2018 г.

DOI: https://doi.org/10.17308/sorpchrom.2018.18/594

Описан теоретический подход и проведена на его основе оценка характеристик селективности неподвижных газохроматографических фаз по структурной формуле фазы. Работа является продолжением развиваемого авторами метода теоретического описания неподвижных фаз в газовой хроматографии. Все параметры, используемые в математических выражениях, имеют физический смысл, переносимы и не требуют проведения дополнительных экспериментов. Сделанные оценки хорошо согласуются с результатами по селективности коммерческих фаз, полученными ранее из экспериментальных данных по индексам Ковача. Подход может быть использован для предсказания поведения аналитов в газовой хроматографии.

Ключевые слова: газовая хроматография, межмолекулярные взаимодействия, неподвижная фаза, полярность, гидрофильность, энергия адсорбции.

\section{Theoretical evaluation of the selectivity characteristics of gas-chromatographic stationary phases}

\author{
Zaitceva E.A., Dolgonosov A.M. \\ Vernadsky Institute of Geochemistry and Analytical Chemistry Russian Academy of Sciences \\ (GEOKHI RAS), Moscow
}

A theoretical approach is described and an evaluation of the selectivity characteristics of the stationary gas-chromatographic phases by the structural phase formula is carried out on its basis. The work is a continuation of the method developed by the authors for describing gas-chromatography stationary phases based on the theoretical method of describing intermolecular interactions in which the total energy of intermolecular interactions is represented in the form of three components: polar, nonpolar forces and hydrogen bond. The proposed method of stationary phases classification differs from existing, mainly theoretical justification, the lack of adjustable parameters and good predictive power; this method does not require special experiments or complex computer calculations, it is very economical to use experimental data. Selectivity is described by two relative characteristics - polarity and hydrophilic; polarity is the ratio of the square of the dipole moment of the stationary phase to its generalized charge and hydrophilicity, in turn, the probability of formation of a hydrogen bond.

Both these characteristics can be calculated from the information on the structural formula of the stationary phase. For most commercial stationary phases, the information on their composition is known, which makes it possible to evaluate the selectivity of these stationary phases, completely abandoning the evaluation experiment. The estimates are in good agreement with the results on the selectivity of commercial phases obtained by us earlier from the experimental data on the Kovacs indices; correctness of the methods is well demonstrated by the selectivity map (Fig. 4). This approach has a good predictive ability and can be used to predict the behavior of analytes in gas chromatography.

Keywords: gas chromatography, intermolecular interactions, stationary phase, polarity, hydrophilicity, adsorption energy. 


\section{Введение}

Современное состояние проблемы описания селективности неподвижных фаз. В настоящее время существует огромное количество жидких неподвижных фаз (НФ) для газовой хроматографии, в том числе одинаковых по селективности, но поразному зашифрованных производителями. Для газохроматографического анализа часто требуется выбор фаз, имеющих различную селективность. Известны традиционные эмпирические методики характеристики фаз, описанные, например, в [1]. Пятимерная схема Роршнайдера [2-5] (и подобные ей методики) основана на предполагаемой независимости пяти видов энергии у пяти эталонных образцов, а расчет схемы строится на системе уравнений с пятью неизвестными, являющимися характеристиками полярности НФ. Однако «чистых» веществ, у которых был бы только один вид взаимодействия не существует; кроме того, независимых видов межмолекулярной энергии не так много - меньше пяти. Отсюда вытекает ошибочность и избыточность схемы, так как пространство параметров Роршнайдера имеет меньшую размерность, а принимающиеся независимыми характеристики эталонных веществ суммируют вклады разных видов энергии и поэтому не могут служить ортами системы координат. Полуэмпирическая модель сольватационных параметров Абрахама [6-7] выглядит более фундаментальной, некоторые ее параметры выводятся теоретически, однако, она еще более сложна в расчетах, чем схема Роршнайдера, а определение всех параметров методом газовой хроматографии невозможно [8-9]. Другие методы используют одномерную схему гидрофобно-гидрофильного баланса [10] где неподвижные фазы (НФ) имеют один оценочный параметр, чего явно недостаточно из-за сложной природы межмолекулярных взаимодействий. В этой связи у НФ с разной селективностью параметры гидрофобно-гидрофильного баланса могут совпадать. Такие схемы обладают ограниченной предсказательной способностью и не могут быть применены к различным экспериментам одинаково. Однако, ввиду отсутствия альтернативных вариантов, подобные методы широко используются для оценки селективности фаз.

Использование теоретических представлений о межмолекулярном взаимодействии (MМВ) для разработки теоретически обоснованного метода характеристики селективности хроматографических фаз является основной темой исследования в работах авторов [11-13]. Развиваемый подход опирается на фундаментальное описание межмолекулярных взаимодействий и лишен недостатков, присущих традиционным методам: не требуются специальные эталонные адсорбаты, отсутствуют математически некорректные процедуры. Показано, что эмпирические данные в форме индексов Ковача могут быть получены из любых, не обязательно специальных экспериментов, а их число может быть сокращено до нескольких (вплоть до двух).

Способ описания межмолекулярного взаимодействия использует общее выражение для энергии: $U=U_{n p}+U_{d p}+n_{H} E_{H}$, где $U_{n p}$ - энергия неполярного взаимодействия - это вандерваальсов потенциал с дисперсионным и отталкивательным членами, например, типа потенциала Леннард-Джонса $[14-15,18], U_{d p}$ - энергия полярного взаимодействия, которая выражается как сумма ориентационной связи Рейнганума-Кеезома и индукционной связи Дебая-Фалькенхагена $[16-17,18], E_{H}$ энергия водородной связи, $n_{H}-$ вероятность образования Н-связи. В развиваемом авторами методе рассматриваются три ключевые характеристики молекул, связанные с поляризуемостью (которая описывается обобщенными зарядами $Q$ ), полярностью (дипольные моменты, $\mu$ ) и способностью к созданию водородных связей (наличие соответствующих атомов и групп, отображаемое параметром $n_{H}$ ). Две по- 
следние характеристики участвуют в так называемых специфических взаимодействиях, которые определяют различия в селективности неподвижных фаз.

Метод позволяет охарактеризовать неподвижные фазы тем же способом, что и молекулы, однако в виду того, что индексы Ковача нормированы на ряд нормальных алканов, они не содержат информацию о неполярном взаимодействии (т.е. обобщенном заряде). В этой связи, в методе используются понятия полярности и гидрофильности, являющиеся отношением характеристик полярных и водородных взаимодействий к характеристике неполярного взаимодействия.

Следует отметить, что теоретические подходы, положенные в основу метода: теория обобщенных зарядов (для определения $U_{n p}$ ), теории Рейнганума-Кеезома и Дебая-Фалькенхагена (для определения $U_{d p}$ ) и квантовомеханическая оценка энергии водородной связи $\left(E_{H}\right),-$ позволяют связать результат совместного действия всех межмолекулярных сил со структурой взаимодействующих молекул. Иными словами, возможен априорный расчет искомых параметров. Такой расчет возможен не только для молекул, но и для хроматографических фаз. В данной работе исследуется такая возможность и описывается первый опыт оценки характеристик селективности НФ без использования данных хроматографических экспериментов.

Для того, чтобы перейти к предмету исследования, дадим предварительно необходимые сведения об обобщенных зарядах и характеристиках селективности фаз.

Сведения из теории обобщенных зарядов. Обобщенные заряды (O3) - это характеристики молекул, которым пропорциональна энергия межмолекулярного неполярного взаимодействия [19-20]:

$$
U_{j j}(r)=Q_{j} Q_{j} u_{b}(r),
$$

где $Q_{j}, Q_{j^{\prime}}$ - обобщенные заряды молекул $j$ и $j^{\prime}, u_{b}(r)$ - функция межмолекулярного расстояния.

Носителями обобщенных зарядов являются жесткие фрагменты молекул; эпитет «жесткий» относится к структуре, объединяющей объекты по принципу обобществления их электронов. Минимальными жесткими фрагментами являются атомы.

Для случая ММВ О3 рассчитывается по формуле:

$$
Q=V^{3 / 4},
$$

где $V$ - электронный объем жесткого фрагмента.

Электронный объем - это число вида:

$$
V=N_{\sigma}+N_{\pi} \sqrt{2}
$$

где $N_{\sigma}, N_{\pi}$ - числа $\sigma$ - и $\pi$-электронов жесткого фрагмента. Электронный объем образован валентными электронами жесткого фрагмента, и для подавляющего числа случаев $N_{\sigma}, N_{\pi}$ - числа $\sigma$ - и $\pi$-электронов, участвующих в ковалентных связях атомов фрагмента, за исключением экранированных, т.е. не попавших в область MMB.

Таким образом, электронный объем жесткого фрагмента вычисляется по формуле (3) как сумма элементарных электронных объемов неэкранированных электронов, а обобщенный заряд молекулы равен сумме О3 нежестко связанных фрагментов, вычисляемых по формуле (2) (в отсутствие нежестких связей вся молекула является жесткой и рассчитывается как жесткий фрагмент).

Обобщенный заряд как фундаментальная характеристика молекулы помимо MМВ участвует в различных свойствах вещества, например, в поляризуемости, величине молекулярной площадки, плотности конденсированной фазы и т.п. 
Метод характеристики селективности НФ по полярности и гидрофильности. С помощью теории обобщенных зарядов было показано, что энергия межмолекулярного взаимодействия в общем случае содержит три независимых члена: первый член - это характеристика неполярной энергии, определяемая обобщенными зарядами; второй член - базирующаяся на известных теориях ориентационного и индукционного взаимодействий характеристика полярной силы, связанная с наличием дипольных моментов хотя бы у одного из взаимодействующих объектов; третий член квантово-химическая оценка энергии водородной связи $[12,13]$. Условием взаимовлияния трех компонент энергии является их зависимость от равновесного расстояния, соответствующего минимуму полной энергии.

В работе [11] предлагается метод характеристики НФ по полярности, который заключается в теоретическом описании межмолекулярных взаимодействий в системе сорбат-сорбент. Мерой полярности НФ является отношение квадрата ее дипольного момента к обобщенному заряду (проще говоря - отношение полярных сил к неполярным):

$$
v_{0}=\frac{\mu_{s p}^{2}}{Q_{s p}},
$$

а мерой гидрофильности отношение вероятности образования Н-связи фазой к ее обобщенному заряду:

$$
w_{0}=\frac{\bar{n}_{H}}{Q_{s p}}
$$

Эти величины выводятся из общего выражения для энергии адсорбции $U_{m, s p}$ молекулы «m» с обобщенным зарядом $Q_{m}$ и дипольным моментом $\mu_{m}$ на НФ (индекс «sp») со стандартной энергией поверхности $U_{0}$ и дипольным моментом $\mu_{s p}$, которые образуют $n_{H}$ водородных связей [11]:

$$
\begin{aligned}
& U_{m, s p}(r)= \\
& =\left[U_{0}\left(Q_{m} f(r)+2 c_{1} \mu_{m}^{2}\right)-\frac{a}{r_{b}^{6} \lambda^{3}} \mu_{s p}^{2}\left(Q_{m}+\frac{c_{2}}{k_{B} T} \mu_{m}^{2}\right)\right]\left(\frac{r_{b}}{r}\right)^{6}-\frac{6 n_{H}}{c_{3}}\left(\frac{r_{b}}{r}\right)^{2}
\end{aligned}
$$

теоретические коэффициенты которой имеют вид:

$$
a=4.948 a_{0}^{3}, c_{1}=\frac{0.9676}{e^{2} a_{0}^{2} \lambda^{3}}, c_{2}=\frac{0.1347}{a_{0}^{3} \lambda^{3}}, c_{3}=\frac{1354 a_{0}}{e^{2}},
$$

где $r_{b}=6.505 a_{0}, a_{0}$ - радиус Бора; $e$ - элементарный заряд; $k_{B} T-$ произведение константы Больцмана на температуру; $\lambda$ - отношение расстояний между центрами диполей и центрами обобщенных зарядов молекул (величина, обычно близкая к единице), $f(r)=2-\left(r / r_{b}\right)^{-6}=2-7.580 \cdot 10^{4}\left(r / a_{0}\right)^{-6}$.

Минимизируя энергию (6) по расстоянию и вводя некоторые замены [13], получаем выражения для расчета параметров селективности НФ - полярности молекулы $v_{i}$, адсорбента $v_{0}$ и параметр гидрофильности адсорбента $w_{0}$, определяемых как

$$
\begin{gathered}
v_{0 i}=\frac{\sqrt{u_{i}}-1-c_{1} v_{i}}{c_{1}\left(1+c_{T} v_{i}\right)} \\
w_{0 i} \approx 0.01523 c_{1} u_{i}^{1 / 3} Q_{i}\left(1+c_{T} v_{i}\right)\left(v_{0 i}-v_{0}\right),
\end{gathered}
$$


где $v=\frac{\mu_{m}^{2}}{Q_{m}} ; v_{0}=\frac{\mu_{s p}^{2}}{Q_{s p}} ; c_{T}=\frac{c_{2}}{k_{B} T}, i-$ индекс молекул, $u_{i}-$ приведенная энергия адсорбции в минимуме для молекулы, $u_{i}=U_{\min } / U_{0} Q_{i}$.

Параметр гидрофильности $w_{0}$ был выведен дополнительно, для более подробного описания НФ. Эта величина представляет собой отношение вероятности образования Н-связи между молекулой и НФ к обобщенному заряду НФ. Следует отметить, что вероятность образования Н-связи, $\overline{n_{H}}$, определяющая гидрофильность фазы, должна быть представлена как произведение вероятности для молекулы $n_{H m}$ (в общем случае не равная 1) и для НФ $n_{H s p}$ :

$$
\overline{n_{H}}=n_{H m} \cdot n_{H s p}
$$

Методика позволяет получить лишь произведение, однако предположения о полноте вероятности образования Н-связи для класса спиртов $\left(n_{H m}=1\right)$ дают возможность найти эти характеристики для неподвижных фаз, а также для других классов молекул.

Таким образом, хотя экспериментальные данные по индексам Ковача не позволяют нам рассчитать обобщенный заряд НФ, относительные характеристики полярности и гидрофильности неподвижных фаз, полученные из экспериментальных данных по газовой хроматографии, могут быть найдены и представлены в виде двумерной карты селективности фаз. На рисунке 1 [13] представлена карта селективности хроматографических фаз, рассчитанная по трем классам молекул - спирты, альдегиды и кетоны и сложные эфиры.

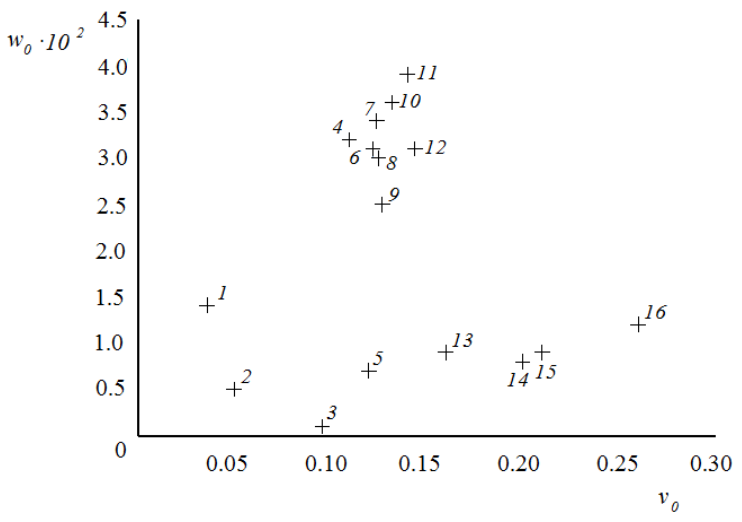

Рис. 1. Карта селективности хроматографических фаз [13] - в координатах полярность $v_{0}\left(\mathrm{D}^{2}\right)$ - гидрофильность $w_{0} \cdot 100$. Фазы представлены в порядке увеличения полярности $v_{0}: 1$ - Сквалан, 2 - SE-30, 3 - OV-7, 4 - Carbowax 6000, 5 - DC-710, 6 - Carbowax 4000, 7 - Carbowax 1540, 8 - Carbowax 20M, 9 - Carbowax 1000, 10 - Carbowax 600, 11 - Carbowax 400, 12 - Carbowax 300, 13 - OV-25, 14 - XE-60, 15 - OV225, 16 - Silar-5CP.

Для экспериментального определения обобщенного заряда НФ, пропорционального стандартному потенциалу поверхности фазы, необходимы данные о временах удерживания или константах Генри. Удобнее было бы рассчитать ОЗ НФ, если известен ее состав. Несмотря на то, что производители шифруют НФ на свое усмотрение, состав большинства самых распространенных фаз известен, вплоть до точной структурной формулы полимера; неизвестным остается лишь состав подготовительной смеси и детергента, используемых при приготовлении хроматографической ко- 
лонки, которые привносят дополнительную полярность и гидрофильность «итоговой» фазе. Однако на данном этапе мы пренебрежем побочными эффектами и покажем, что априорный расчет характеристик фаз, во-первых, возможен, и, во-вторых, он согласуется с разработанной ранее методикой экспериментального получения этих характеристик.

\section{Теоретическая часть}

Расчет обобщенного заряда НФ. Если в общем выражении для энергии взаимодействия, $U(r)=Q_{j} Q_{j^{\prime}} u_{b}(r)$, где $u_{b}(r)=e^{2} / a_{0}\left[1.938 \cdot 10^{5}\left(r / a_{0}\right)^{-12}-5.115\left(r / a_{0}\right)^{-6}\right]$, выделить потенциал поля поверхности макроскопического тела $U_{A}(r)=Q_{s p} u_{b}(r)$, то выражение для энергии молекулы с обобщенным зарядом, равным $Q$, в этом поле запишется в виде $U=Q U_{A}(r)$. Подстановка в предыдущее выражение равновесного расстояния дает значение стандартной энергии поверхности $U_{0}=Q_{s p} u_{b, m}=-0.08854 Q_{s p}$ (кДж/моль), где $Q_{s p}-$ обобщенный заряд, рассчитанный для полусферического объема, выходящего на поверхность однородной конденсированной фазы, с радиусом, равным радиусу экранирования валентных электронов вещества этой фазы [21].

Радиус экранирования электрона - это максимальное удаление электрона, входящего в обобщенный заряд, от места взаимодействия фрагмента. Он определяется по формуле:

$$
r_{s} \approx e \sqrt{\frac{6 a_{0}}{E_{1}}}
$$

где $E_{1}$ - энергия наименее сильной одинарной ковалентной связи данного атома. Если измерять расстояние в нм, а энергию в кДж/моль, то получим формулу $r_{s}=6.642 E_{1}^{-1 / 2}$, согласно которой для энергий в интервале 250-500 кДж/моль следуют значения радиуса в области 0.3-0.4 нм.

Величина $Q_{s p}$ является максимальной при сканировании поверхности. При фиксированном значении электронного объема $\sum V_{j}$, диктуемом радиусом экранирования, максимум величины $Q_{s p}=N Q=N\left(\sum V_{j} / N\right)^{3 / 4}$ получим при равенстве вкладов всех заходящих в полусферу экранирования молекул: $V_{0}=\frac{1}{N} \sum V_{j}=V_{j}=$ const и при наибольшем числе этих молекул $N$. Итак, величина $Q_{s p}$ определяется по формуле:

$$
Q_{s p}=N V_{0}^{3 / 4}=\alpha N Q \text {, }
$$

коэффициент $\alpha \geq 1$,

$$
\alpha=\left[V_{0} /\left(V_{0}-s\right)\right]^{3 / 4}=\left(Q^{3 / 4}+s\right)^{3 / 4} / Q,
$$

$Q$ - обобщенный заряд характерного фрагмента молекулы НФ (мономера), $s$ - число экранированных электронов.

В изотропной среде можно считать, что отрезок, соединяющий центры молекул, перпендикулярен к разделяющей их плоскости (см., напр., рис. 2). Проекция молекулы на эту плоскость называется молекулярной площадкой. Как было найдено в [19], молекулярная площадка единичной молекулы $w$ выражается через ее обобщенный заряд:

$$
w=9.939 a_{0}^{2} Q=0.0278 Q \mathrm{HM}^{2}
$$




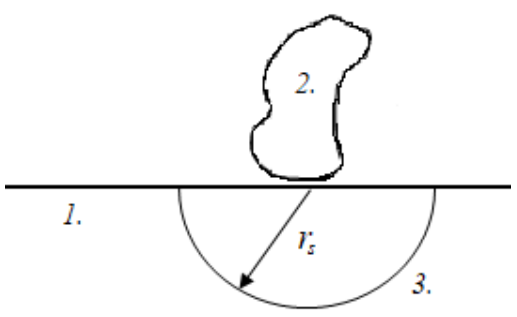

Рис. 2. Схематичное изображение области взаимодействия молекулы адсорбата с поверхностью НФ. 1 - поверхность НФ, 2 - молекула адсорбата, 3 - полусфера экранирования, $r_{s}$ - радиус экранирования.

В работе [21] показано, что объем молекулы равен объему прямого цилиндра с основанием в виде молекулярной площадки:

$$
W=w r_{b}=64.64 a_{0}^{3} Q=9.57 \cdot 10^{-3} Q \mathrm{Hм}^{3}
$$

Число молекул, помещающихся в полусферу экранирования, найдем, поделив объем $W_{s}$ на объем, приходящийся на молекулу с учетом плотности укладки:

$$
N=\kappa \frac{W_{s}}{W}=\frac{2 \pi \kappa e^{3}\left(6 a_{0}\right)^{3 / 2}}{3 \cdot 64.64 a_{0}^{3} Q E_{1}^{3 / 2}}=\frac{1}{3 Q}\left(\frac{e^{2}}{a_{0} E_{1}}\right)^{3 / 2},
$$

откуда согласно (11):

$$
Q_{s p} \equiv \alpha N Q=\frac{\alpha}{3}\left(\frac{e^{2}}{a_{0} E_{1}}\right)^{3 / 2}
$$

Расчет полярности и гидрофильности НФ. Рассчитать полярность и гидрофильность НФ, зная ее обобщенный заряд можно по формулам (4) и (5), соответственно: $v_{0}=\frac{\mu_{s p}^{2}}{Q_{s p}} ; w_{0}=\frac{\bar{n}_{H}}{Q_{s p}}$.

Дипольный момент фазы определяется по характерному молекулярному фрагменту из справочных данных или рассчитывается с помощью программы Chem3D [22]. Фрагмент НФ выбирается таким образом, чтобы максимально отразить свойства молекулы фазы - мономерное звено и функциональная группа, в случаях, когда НФ содержит несколько различных функциональных групп, необходимо включить в этот фрагмент все функциональные группы, даже неполярные (например, фенильные заместители в полисилоксане). При использовании программы получают наиболее энергетически выгодную конформацию молекулы и рассчитывают ее дипольный момент. Найденное значение дипольного момента используется в расчете полярности НФ по формуле (4).

Значение вероятности образования Н-связи равное 1 свойственно целому ряду соединений, например, спиртам и карбоновым кислотам. Ранее [13] было выявлено, что сложные эфиры с коротким радикалом способны образовывать Н-связь с определенной долей вероятности. Для расчета теоретического значения гидрофильности НФ необходимо учесть, что вклад в гидрофильность будут вносить только функциональные группы, способные образовывать Н-связь.

Вероятность образования Н-связи $n_{H}$ может быть найдена из структурной формулы и состава НФ. Вся фаза рассматривается как однородная поверхность, на которой в зависимости от структурной формулы находятся все атомы молекул НФ. Но, например, в случае метилсилоксановых фаз, распределение функциональных групп не может быть «равномерным» (имеется ввиду, что функциональные группы 
могут быть не в каждой сфере экранирования) из-за малого их содержания. Получается, что какие-то полусферы экранирования будут содержать только метильные радикалы, а какие-то только функциональные группы - в одном случае Н-связь не образуется, а во втором с высокой долей вероятности образуется, или содержать и те, и другие группы, что будет означать возможность образования Н-связи, но с меньшей вероятностью. Для оценки гидрофильности такой неоднородной или смешанной НФ рассматривается средняя величина вероятности. Например, в случае полисилоксановых фаз гидрофильность рассчитывается в зависимости от процента содержания полярных групп, метильные или фенильные заместители Н-связи с аналитами не образуют. Общая гидрофильность смеси фаз рассчитывается по формуле (5) при подстановке:

$$
n_{H s p}=\sum_{i} k_{i} \cdot n_{H i},
$$

где $k_{i}$ - объемная доля компонента фазы, $n_{H}$ - вероятность образования Н-связи для компонента фазы. Формула, аналогичная (17), справедлива и для расчета квадрата дипольного момента сложной смеси НФ.

\section{Обсуждение результатов}

Расчет характеристик НФ проводится в несколько этапов:

- выбор фрагмента НФ, который максимально отображает свойства фазы; также можно подобрать максимально близкое по структурной формуле к НФ соединение, которое бы отражало свойства этой фазы;

- для выбранного фрагмента рассчитывается О3 по формуле (14), расчет средней энергии $E_{1}$ через радиус экранирования подробно представлен в [21], коэффициент $\alpha=\left(\frac{V_{0}}{V_{0}-s}\right)^{3 / 4}=\frac{\left(Q^{4 / 3}+s\right)^{3 / 4}}{Q} \approx 1$ (большие значения $\alpha$, но не больше 1.4 , свойственны кремнийсодержащим соединениям из-за большого размера атома $\mathrm{Si}$ ). В таблице 1 представлены параметры для расчета О3;

Таблица 1. Расчет О3 фрагмента НФ

\begin{tabular}{|c|c|c|c|}
\hline Название НФ & $\alpha$ & $E_{1}$, кДж/моль & $Q_{s p}$ \\
\hline Сквалан & 1.06 & 340 & 10.14 \\
\hline SP-2250 & 1.19 & 374 & 10.04 \\
\hline SP-2340 & 1.10 & 444 & 12.07 \\
\hline Carbowax 20M & 1 & 338 & 8.90 \\
\hline CW-20M & 1 & 338 & 8.90 \\
\hline SE-30 & 1.07 & 366 & 6.40 \\
\hline DC-710 & 1.19 & 374 & 10.04 \\
\hline XE-60 & 1.09 & 395 & 10.20 \\
\hline OV-225 & 1.09 & 367 & 11.45 \\
\hline Silar-5CP & 1.2 & 381 & 11.40 \\
\hline
\end{tabular}

- в программе Chem3D строится выбранный фрагмент HФ, рассчитывается дипольный момент. Например, для ПЭГ фаз фрагментом структурной формулы будет являться мономер -O- $\mathrm{CH}_{2}-\mathrm{CH}_{2}$ - или диэтиловый эфир. При расчете дипольного момента НФ необходимо учитывать число атомов кислорода в полусфере экраниро- 
вания: $n_{o}=\frac{2 \pi r_{s}^{3} N_{A} \rho}{3 m_{1}}$, где $N_{A}-$ число Авогадро, $\rho$ - плотность НФ, $m_{1}$ - молекулярная масса мономера НФ. Тогда можно рассчитать с помощью программы дипольный момент большого фрагмента НФ, а затем сравнить его с фрагментом сферы экранирования; получится более точный расчет по всей фазе. На рисунке 2 схематично представлена область взаимодействия молекулы адсорбата с поверхностью НФ - полукругом выделена полусфера экранирования. Отметим, что селективность хроматографического удерживания определяется взаимодействием молекул фазы и аналита, поэтому форма поверхности контакта, различающая случаи адсорбции и растворения, несущественна;

- рассчитывается полярность - $v_{0}$ - неподвижной фазы (4). В таблице 2 представлены величины дипольного момента фаз и полярности;

Таблица 2. Расчет дипольных моментов (D) и полярности $\mathrm{HФ}\left(\mathrm{D}^{2}\right)$ с учетом числа атомов кислорода $n_{O}$ в полусфере экранирования НФ

\begin{tabular}{|c|c|c|c|}
\hline \multirow{2}{*}{ Название НФ } & $n_{O}$ & $\begin{array}{c}\text { Дипольный момент } \\
\mu_{s p}\end{array}$ & $v_{0}$ \\
\hline Сквалан & 0 & 0 & 0 \\
\hline SP-2250 & 0.27 & 0.365 & 0.013 \\
\hline SP-2340 & 1.65 & 1.850 & 0.28 \\
\hline Carbowax 20M & 1.1 & 1.063 & 0.13 \\
\hline CW-20M & 1.1 & 1.132 & 0.13 \\
\hline SE-30 & 0.69 & 0.125 & 0.002 \\
\hline DC-710 & 0.27 & 0.365 & 0.013 \\
\hline XE-60 & 0.93 & 1.680 & 0.28 \\
\hline OV-225 & 0.825 & 1.710 & 0.26 \\
\hline Silar-5CP & 0.96 & 1.810 & 0.29 \\
\hline
\end{tabular}

- рассчитывается вероятность образования Н-связи (17); стоит отметить, что не все фазы способны образовывать Н-связь, необходимо наличие электроотрицательных атомов, способных к Н-связыванию;

- рассчитывается гидрофильность - $w_{0}-$ НФ (5). В таблице 3 представлены рассчитанные характеристики вероятности образования Н-связи неподвижной фазой и гидрофильность НФ.

Таблица 3. Вероятность образования Н-связи и гидрофильность НФ.

\begin{tabular}{|c|c|c|}
\hline Название НФ & $n_{H}$ & $w_{0}$ \\
\hline Сквалан & 0 & 0 \\
\hline SP-2250 & 0.19 & 0.019 \\
\hline SP-2340 & 1.00 & 0.083 \\
\hline Carbowax 20M & 0.27 & 0.030 \\
\hline CW-20M & 0.27 & 0.030 \\
\hline SE-30 & 0 & 0 \\
\hline DC-710 & 0.19 & 0.019 \\
\hline XE-60 & 0.25 & 0.025 \\
\hline OV-225 & 0.25 & 0.022 \\
\hline Silar-5CP & 0.50 & 0.044 \\
\hline
\end{tabular}

На рисунке 3 представлены структурные формулы некоторых НФ. Данные фазы могут быть нанесены на стенки хроматографической колонки в чистом виде 
или в виде смесей - например, различные силоксановые фазы получаются путем смешивания двух или нескольких силоксановых НФ с различными заместителями или без них, примером такой фазы здесь является НФ ХЕ-60 - она получается путем смешивания чистого полидиметилсилоксана с 50\%-цианоэтил-50\%-метилсилоксаном в пропорции $1: 1$.

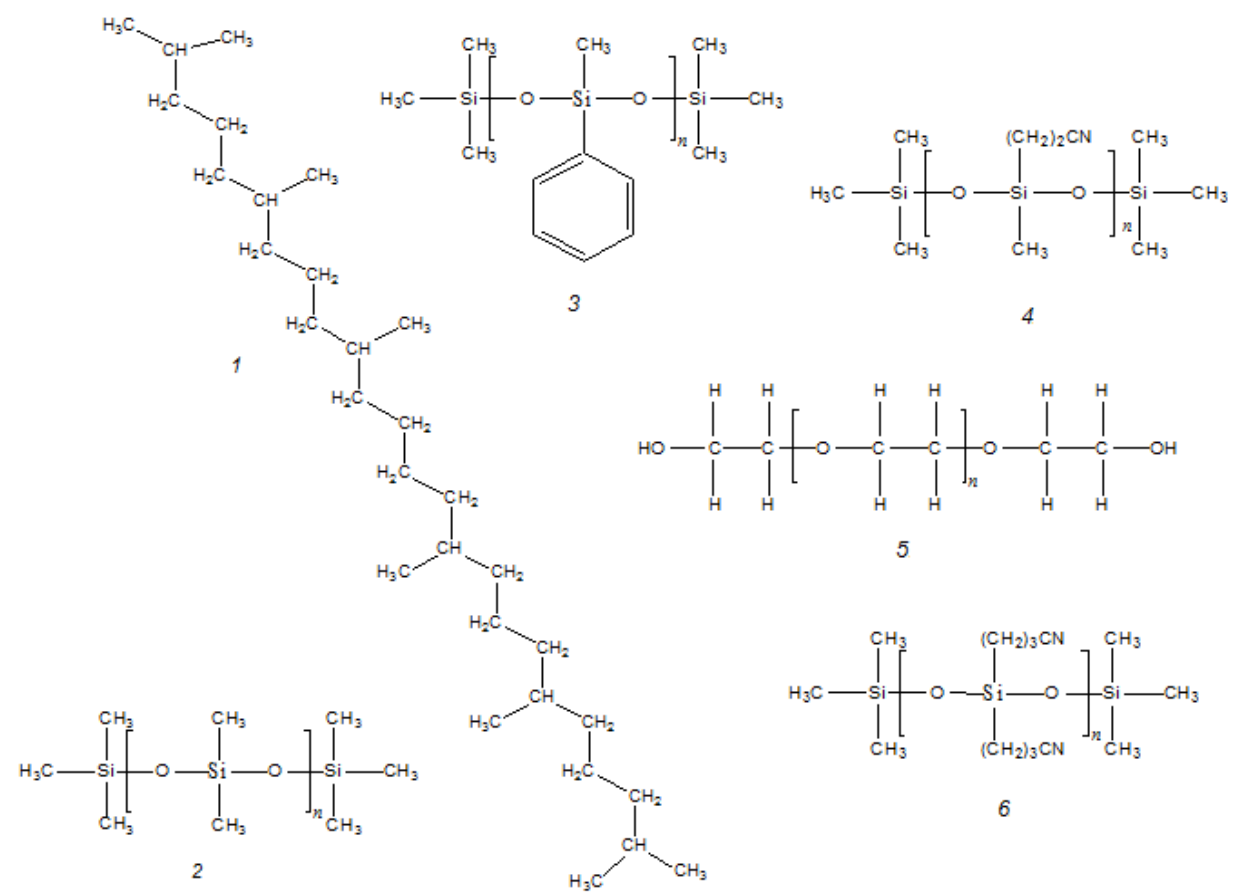

Рис. 3. Структурные формулы некоторых неподвижных фаз: 1 - сквалан, 2 - полидиметилсилоксан, 3 - 50\%-фенил-50\%-метилсилоксан, 4 - 50\%-цианоэтил$50 \%$-метилсилоксан, 5 - полиэтиленгликоль, 6 - цианопропилсилоксан.

В случае ПЭГ-фаз $n_{H}$ будет зависеть, главным образом, от наличия на поверхности атомов кислорода в мономере [-O- $\left.\mathrm{CH}_{2}-\mathrm{CH}_{2}-\right]$, т.к. функциональных групп (-OH) на одну молекулу всего 2, а молекулярная масса молекулы равна 20000, следовательно, участием гидроксильных групп в образовании Н-связи можно пренебречь. Поверхность НФ рассматривается как равномерный слой ПЭГ и необходимо рассчитать, какое количество атомов будет находиться на поверхности НФ в пределах радиуса экранирования. Объем, приходящийся на 1 атом кислорода равен объему мономера $m_{1} /\left(N_{A} \rho\right)$ (мономеры содержат по одному атому кислорода). Число атомов кислорода в полусфере экранирования радиусом $r_{s}$ равно: $n_{o}=1.779$.

Для нахождения вероятности $n_{H}$ необходимо оценить не только количество атомов кислорода, входящих в полусферу экранирования, но и вероятность нахождения атома кислорода в правильной конформации - неподеленной парой электронов вовне поверхности НФ. Эта вероятность и будет вероятностью образования Нсвязи неподвижной фазой, потому что другие атомы, оказавшиеся в неправильной конформации не смогут участвовать во взаимодействии из-за строгого геометрического условия образования Н-связи, связанного с ее узкой направленностью [25]. Случайное расположение полимера вблизи поверхности фазы приводит к шести равновероятным конформациям, различающимся положением неподеленной пары атома кислорода - по числу пространственных направлений. Вероятность нахожде- 
ния в полусфере экранирования атома кислорода в одном из 6 положений, удобном для образования Н-связи, равна: $n_{\text {Hsp }}=n_{o} / 6=\frac{3.14 \cdot 407 \cdot 10^{-23} \cdot 6.02 \cdot 10^{23}}{9 \cdot 44}=0.27$.

В таблице 4 представлены рассчитанные двумя способами характеристики НФ - расчет через индексы Ковача аналитов и расчет по структурной формуле НФ. На рисунке 4 представлена карта селективности НФ, рассчитанная по реперным молекулам шкалы полярности Роршнайдера-МакРейнольдса. Значения индексов Ковача имеют слабую зависимость от температуры, поэтому, для большей точности, при расчете характеристик НФ из индексов Ковача использовались данные, полученные при одной температуре $-120^{\circ} \mathrm{C}(393 \mathrm{~K})$ для всех фаз. Определяемые характеристики от температуры не зависят и, соответственно, для априорного расчета, температура не учитывается.

Таблица 4. Сравнение экспериментально рассчитанных характеристик НФ (по методике, описанной в [11-12]) и априорного расчета характеристик селективности НФ

\begin{tabular}{|c|c|c|c|c|c|c|}
\hline Название НФ & Состав НФ & $n_{H}$ & $v_{0}$ & $v_{0}()^{*} *$ & $w_{0}$ & $w_{0}(')$ \\
\hline Сквалан & $\begin{array}{c}2,6,10,15,19,23- \\
\text { гексаметилтетракозан }\end{array}$ & 0 & 0 & 0.006 & 0 & 0.013 \\
\hline SP-2250 & $\begin{array}{c}\text { 50\%-фенил-50\%- } \\
\text { метилсилоксан }\end{array}$ & 0.19 & 0.013 & 0.014 & 0.019 & 0.032 \\
\hline SP-2340 & Цианопропилсилоксан & 1.00 & 0.28 & 0.35 & 0.087 & 0.102 \\
\hline Carbowax 20M & $\begin{array}{l}\text { Полиэтиленгликоль } \\
\text { (MW } 20 \text { 000) }\end{array}$ & 0.27 & 0.13 & 0.13 & 0.030 & 0.031 \\
\hline CW-20M & $\begin{array}{c}\text { Полиэтиленгликоль } \\
\text { (MW } 20 \text { 000) }\end{array}$ & 0.27 & 0.13 & 0.14 & 0.030 & 0.031 \\
\hline SE-30 & Полидиметилсилоксан & 0 & 0.002 & 0.05 & 0.001 & 0.002 \\
\hline DC-710 & $\begin{array}{c}50 \% \text {-фенил-50\%- } \\
\text { метилсилоксан }\end{array}$ & 0.19 & 0.013 & 0.012 & 0.019 & 0.023 \\
\hline XE-60 & $\begin{array}{c}25 \% \text {-цианоэтил-75\%- } \\
\text { метилсилоксан }\end{array}$ & 0.25 & 0.28 & 0.20 & 0.025 & 0.028 \\
\hline OV -225 & $\begin{array}{c}\text { 25\%-цианопропил 25\%- } \\
\text { фенил-50\%- } \\
\text { метилсилоксан }\end{array}$ & 0.25 & 0.26 & 0.21 & 0.022 & 0.011 \\
\hline Silar-5CP & $\begin{array}{c}\text { 50\%-цианопропил-50\%- } \\
\text { фенилсилоксан }\end{array}$ & 0.50 & 0.29 & 0.26 & 0.044 & 0.049 \\
\hline
\end{tabular}

* апострофом отмечены экспериментальные значения.

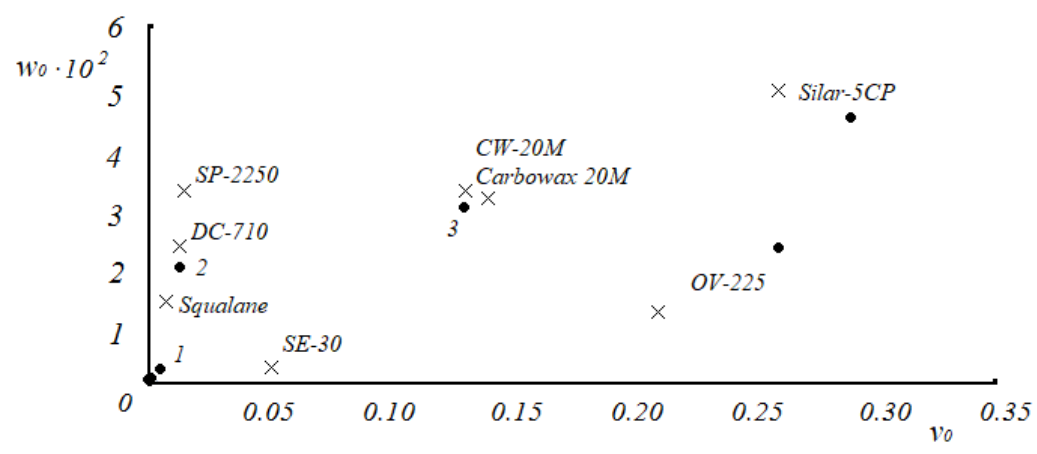

Рис. 4. Карта селективности НФ, полученная при расчете характеристик НФ из индексов Ковача (крестики) и априорном расчете (точки). 1 - близкое к нулю значение полярности и гидрофильности фазы SE-30 и нулевые значения характеристик для Сквалана, 2 - совпадающиеся значения полярности и гидрофильности фаз DC-710 и SP-2250, 3 - совпадающие значения характеристик для ПЭГ-фаз Carbowax 20M и CW-20M. 
Сильное расхождение экспериментальных и теоретических значений наблюдается у Сквалана и SE-30. Согласно теории, обе фазы являются абсолютно неполярными - Сквалан по шкале Роршнайдера-МакРейнольдса считается эталонной неполярной фазой - не способными образовывать Н-связь, а расчет по экспериментальным данным показывает ненулевые полярность, в случае SE-30, или гидрофильность, в случае сквалана. Это связано, как уже говорилось ранее, со способом приготовления колонки, с влиянием остаточных количеств детергента и растворителя. Их учет в априорном расчете осложнен недостаточностью информации об их использовании.

Стоит обратить внимание на одинаковые по составу фазы. Например, НФ, зашифрованные как Carbowax-20M и CW-20M, имеют одинаковый состав; значения экспериментальных данных по их полярности и гидрофильности достаточно близки, теоретические расчеты, соответственно, совпадают. SP-2250 и DC-710 также одинаковые по составу фазы, но экспериментальные данные показывают некоторое различие из-за разных условий приготовления колонок и разделения.

Отдельно надо выделить фазы XE-60, OV-225 и Silar-5CP, у которых и теоретические и экспериментальные значения полярности и гидрофильности растут с увеличением количества циан-заместителей, что согласуется с уравнением (17), расхождение данных достаточно мало.

НФ SP-2340 обладает максимальной вероятностью образования Н-связи, это связано с тем, что атом азота всегда будет находиться в правильной конформации на поверхности фазы, в отличие от кислорода в ПЭГ-фазах. В этой НФ все метильные радикалы замещены на цианопропильные группы, соответственно, фаза не «разбавляется» и значение $n_{H}$ будет равным 1.

\section{Заключение}

Таким образом, разработан способ априорного расчета параметров селективности неподвижных фаз для газовой хроматографии, который базируется на теоретическом подходе, включающем в себя: описание неполярных взаимодействий с помощью теории обобщенных зарядов, полярных взаимодействий по известным описаниям ориентационных и индукционных сил и водородных связей, существование которых лимитируется квантовомеханическими ограничениями. Исходными данными для расчета являются сведения о молекулярном строении НФ.

Полученные в результате априорного расчета характеристики фаз удовлетворительно согласуются с характеристиками, найденными авторами ранее из экспериментальных данных по индексам удерживания [11-13], тем самым подтверждая правильность предложенной там методики построения карты селективности фаз. Разработанный способ дает возможность сравнивать неподвижные фазы не только между собой, но и с молекулами разделяемой смеси.

Работа выполнена при финансовой поддержке РФФИ (грант 18-03-00382a).

\section{Список литературы}

1. Rohrschneider L. // J.Chomatogr. 1965. 3. ороль А.Н. Неподвижные фазы в гаVol. 17. pp. 1-12

2. McReynolds W.O. // J.Chromatogr. М. Химия. 1985. 240 c. 1970. Vol. 8. P. 685. pp. 337-345. 
4. McReynolds W.O. Gas Chromatographic Retention Data. Preston. Technical Abstracts Co. Niles. 1966. 335 p.

5. Лурье А.А. Сорбенты и хроматографические носители (справочник). М. Химия. 1972. $320 \mathrm{c}$.

6. Poole C.F., Poole S.K. // J. Chromatogr. A. 2002. Vol. 965. pp. 263-299.

7. Abraham M.H., Du C.M., Platts J.A. // J. Org. Chem. 2000. Vol. 65. pp. 7114-7118.

8. Lombardo F., Shalaeva M.Y., Tupper K.A., Gao F. et al. // J. Med. Chem. 2000. Vol. 43. pp. 2922-2928.

9. Dias N.C., Poole C.F. // J. Planar. Chromatogr. 2000. Vol. 13. pp. 337-347.

10. Долгоносов А.М., Рудаков О.Б., Суровцев И.С., Прудковский А.Г. Колоночная аналитическая хроматография как объект математического моделирования. ГЕОХИ РАН. Воронежский ГАСУ. Воронеж. 2013. 400 c.

11. Долгоносов А.М., Зайцева Е.А. // Сорбиионные и хроматографические проuессыл. 2014. Т. 14. № 4. С. 578-590.

12. Долгоносов А.М., Зайцева Е.А. // Сорбиионные и хроматографические проиессы. 2015. Т. 15. № 3. С. 321-332.

13. Долгоносов А.М., Зайцева Е.А. // Вестник Университета Дубна. 2015. № 1. С. 36-41.

14. Reinganum M. // Ann.d.Physik. 1912. Vol. 38. P. 649-668.

15. Keesom W.H. // Phys.Z. 1921. Vol. 22. pp. 129-141.

\section{References}

1. Rohrschneider L., J.Chomatogr., 1965, Vol. 17, pp. 1-12.

2. McReynolds W.O., J.Chromatogr., 1970 Vol. 8, pp. 337-345.

3. Korol' A.N. Nepodvizhnye fazy v gazozhidkostnoi khromatografii: Spravochnik. M., Khimiya, 1985, 240 p.

4. McReynolds W.O., Gas Chromatographic Retention Data. Preston, Technical Abstracts Co, Niles, 1966, 335 p.

5. Lur'e A.A. Sorbenty i khromatograficheskie nositeli (spravochnik), M., «Khimiya», 1972, $320 \mathrm{p}$.

6. Poole C.F., Poole S.K., J. Chromatogr. A, 2002, Vol. 965, pp. 263-299.

7. Abraham M.H., Du C.M., Platts J.A., J. Org. Chem., 2000, Vol. 65, pp. 7114-7118.
16. Debye P. // Phys.Z. 1920. Vol. 21. pp. 178-187.

17. Falkenhagen M. // Phys.Z. 1922. Vol. 23. pp. 87-95.

18. Каплан И.Г. Межмолекулярные взаимодействия. Физическая интерпретация, компьютерные расчеты и модельные потенциалы. М. БИНОМ. Лаборатория знаний. 2012. 394 c.

19. Долгоносов А.М. Модель электронного газа и теория обобщенных зарядов для описания межатомных взаимодействий и адсорбции. М. ЛИБРОКОМ. 2009. 167 с.

20. Долгоносов А.М. // Журн. физ.химии. 2001. Т. 75. № 10. С.1813-1820.

21. Долгоносов А.М. // Известия Академии наук. Серия химическая. 2016. № 4. С. 952-963.

22. Cambridge soft - chemical drawing, Chemical databases, Enterprises solutions, Desktop software, Consulting services. Available at: http://www.cambridgesoft.com accessed 1 July2018.

23. Chunhui Lu, Weimin Guo, Chunsheng Yin. // Analytica Chimica Acta. 2006. Vol. 561. pp. 96-102.

24. Dannenberg J.J., Haskamp L., Masunov A. // J. Phys. Chem. A. 1999. Vol. 103. pp. 7083-7086.

25. Бушуев Ю.Г. Дисс. докт.хим.наук. Иваново. 2001. 345 с.

26. Пецев Н., Коцев Н. Справочник по газовой хроматографии. М. Мир. 1987. 260 с.

8. Lombardo F., Shalaeva M.Y., Tupper K.A., Gao F. et al., J. Med. Chem., 2000, Vol. 43, pp. 2922-2928.

9. Dias N.C., Poole C.F.., J. Planar. Chromatogr., 2000, Vol. 13, pp. 337-347.

10.Dolgonosov A.M., Rudakov O.B., Surovtsev I.S., Prudkovskii A.G. Kolonochnaya analiticheskaya khromatografiya kak ob' ekt matematicheskogo modelirovaniya. GEOKHI RAN Voronezhskii GASU, Voronezh, 2013, 400 p.

11.Dolgonosov A.M., Zaitseva E.A., Sorbtsionnye i khromatograficheskie protsessy, 2014, Vol. 14, No 4, pp. 578-590.

12.Dolgonosov A.M., Zaitseva E.A., Sorbtsionnye $i$ khromatograficheskie protsessy, 2015, Vol. 15, No 3, pp. 321-332. 
13.Dolgonosov A.M., Zaitseva E.A., Vestnik Universiteta Dubna, 2015, No 1, pp. 36-41.

14.Reinganum M., Ann.d.Physik, 1912, Vol. 38, pp. 649-668.

15.Keesom W.H., Phys.Z., 1921, Vol. 22, pp. 129-141.

16.Debye P., Phys.Z., 1920, Vol. 21, pp. 178187.

17.Falkenhagen M., Phys.Z., 1922, Vol. 23, pp.87-95.

18.Kaplan I.G. Mezhmolekulyarnye vzaimodeistviya. Fizicheskaya interpretatsiya, komp'yuternye raschety i model'nye potentsialy, M., BINOM, Laboratoriya znanii. 2012, pp. 394.

19.Dolgonosov A.M. Model' elektronnogo gaza i teoriya obobshchennykh zaryadov dlya opisaniya mezhatomnykh vzaimodeistvii $\mathrm{i}$ adsorbtsii, M., LIBROKOM, 2009, 167 p.

Зайцева Елена Александровна - аспирантка второго года обучения, лаборатория сорбционных методов, Институт геохимии и аналитической химии им. В.И. Вернадского РАН (ГЕОХИ РАН), Москва

Долгоносов Анатолий Михайлович - д.Х.н., ведущий научный сотрудник, лаборатория сорбционных методов, Институт геохимии и аналитической химии им. В.И. Вернадского РАН (ГЕОХИ РАН), Москва
20.Dolgonosov A.M., Zhurn. fiz. khimii, 2001, Vol. 75, No 10, pp.1813-1820.

21.Dolgonosov A.M., Izvestiya Akademii nauk. Seriya khimicheskaya, 2016, No 4, pp. 952-963.

22. Cambridge soft - chemical drawing, Chemical databases, Enterprises solutions, Desktop software, Consulting services. Available at: http://www.cambridgesoft.com (accessed 1 July 2018).

23. Chunhui Lu, Weimin Guo, Chunsheng Yin., Analytica Chimica Acta, 2006, Vol. 561, pp. 96-102.

24.Dannenberg J.J., Haskamp L., Masunov A., J. Phys. Chem. A, 1999, Vol. 103, pp. 70837086.

25.Bushuev Yu.G. Diss. dokt.khim.nauk, Ivanovo, 2001, pp. 345.

26.Petsev N., Kotsev N. Spravochnik po gazovoi khromatografii, M., Mir, 1987, 260 p.

Zaytseva Elena A. - PhD student, Lab of Sorption Methods, Vernadsky Institute of Geochemistry and Analytical Chemistry Russian Academy of Sciences (GEOKHI RAS), Moscow., lil-dante@mail.ru

Dolgonosov Anatoly M. - Dr.Sci.(Chem.), Leading scientific researcher, Lab of Sorption Methods, Vernadsky Institute of Geochemistry and Analytical Chemistry Russian Academy of Sciences (GEOKHI RAS), Moscow, amdolgo@mail.ru 